\title{
A Novel Technique for Transmission of M-Ary Signal through Wireless Fading Channel Using Wavelet Denoising
}

\author{
Md. Zahangir Alam, ${ }^{1}$ Charles Chittaranjan Patra, ${ }^{2}$ Colin Patra, ${ }^{2}$ and M. Abdus Sobhan ${ }^{3}$ \\ ${ }^{1}$ EEE Department, ADUST, Dhaka, Bangladesh \\ ${ }^{2}$ WebSatMedia Pte. Ltd., Technopark, Singapore 469003 \\ ${ }^{3}$ School of Engineering and Computer Science, IUB, Dhaka, Bangladesh
}

Correspondence should be addressed to Md. Zahangir Alam, ripon83.ru@gmail.com

Received 30 March 2011; Accepted 24 June 2011

Academic Editor: Youyun Xu

Copyright (c) $2011 \mathrm{Md}$. Zahangir Alam et al. This is an open access article distributed under the Creative Commons Attribution License, which permits unrestricted use, distribution, and reproduction in any medium, provided the original work is properly cited.

The paper proposes a novel technique for reducing noise in M-ary signal transmission through wireless fading channel using wavelet denoising that play the key role. The paper also explains that the conventional threshold-based technique is not capable of denoising M-ary quadrature amplitude modulated (M-QAM) signals having multilevel wavelet coefficients through wireless fading channels. A detailed step by step wavelet decomposition and reconstruction processes are discussed here to transform a signal function into wavelet coefficients using simulation software like MATLAB. A 16-QAM modulated symbol through a Rician fading channel is weighted by a control variable of complex form to force the mean of each detail coefficient except low frequency component to zero to enhance noiseless property. The bit error rate (BER) performance of the simulation results are furnished to show the effectiveness of the proposed technique. The root mean square of the deviation of the reconstruct signal from the original signal is used to express the effectiveness of the proposed technique. The traditional denoising provides very high value (above $90 \%$ ) of the percentage root mean square difference (PDR) and the proposed technique provides only 10\% PDR value for the symbol through a noisy channel. The result of the simulation study reveals that the BER performance can be increased using an appropriate control variable to force the mean of each detail coefficient to zero.

\section{Introduction and Literature Review}

The signal transmitted through a wireless channel may arrive at the receiver through a number of paths with different amplitude, phase, and time delay due to multipath fading. The relative movement of either transmitter or receiver or both causes time varying multipath fading. Equalizer is used to minimize the fading effect, but the design complexity of the equalizer increases with the increase of data rate. Wavelet decomposition is in use in the area of image processing, image compression, segmentation, and denoising [1-5]. The wavelet coefficients are estimated from the noisy signals using maximum a posteriori (MAP) estimator [6]. In [6], four new bivariate distributions are proposed to wavelet coefficients of natural images to calculate the dependency between a coefficient and its parent. The wavelet denoising algorithm is extremely useful to reconstruct the original image signal from its noisy form. The main idea of denoising is to subtract the threshold value $T$ from all coefficients greater than the threshold one and set other coefficients to zero [7]. The wavelet transform of a signal is passed through a threshold that removes the coefficients below a certain value. Most of the energy of a signal is associated in the lowfrequency region, and consequently the approximate coefficients are also contained in this region. Thus the highfrequency noise terms [8] are removed through this process. Wavelet denoising based on threshold scheme can be found in [9-11]. Wavelet soft-thresholding algorithm has been proposed for image denoising to reconstruct a noisy image in [12]. Fractal image denoising method is used to generate an original image from the noisy one discussed in [13]. A robust wavelet denoising-based estimator using a robust loss function is used to denoise a wireless signal through a Gaussian noisy channel [14]. Traditional wavelet denoising 
uses a threshold value [7] to reconstruct noisy wavelet coefficients by allowing actual noise coefficient if greater than the threshold and zero otherwise.

In this paper, the authors attempt to apply wavelet decomposition technique to generate wavelet coefficients of a noisy signal passing through a fading channel. The multipath fading channel has been modeled recently as reported in [15]. The reconstruction of the original symbol from the noisy transmitted symbol through the fading channel using wavelet denoising technique is discussed in detail in this paper. Wavelet decomposition and reconstruction methods are also derived in [16-18]. The wavelet decomposition and reconstruction techniques are discussed here to generate wavelet coefficients for a noisy symbol and reconstruct the signal from the coefficients by a computer simulation using a programming language like C, Java, or software like MATLAB. The threshold-based denoising technique is used to reconstruct the modulated symbol. The problem of the traditional denoising is that the wavelet coefficients of 16-QAM or 64-QAM modulated symbols consist of multiple-bits and the thresholding technique is not able to estimate the noisy coefficients either greater or less than the threshold value. The authors also propose, in this paper, a denoising technique for multiple-bits symbol using a complex weighting control variable to force the mean of each high-frequency detail coefficient to zero. The transmitted 16-QAM symbol through a Rician fading channel is received in MATLAB simulation environment. The received noisy symbol is transformed to generate wavelet coefficients based on the wavelet decomposition technique. The proposed $\mathrm{M}$-ary denoising technique predicts the actual detail coefficients from the received noisy coefficients. The transmitted signal is reconstructed from the predicted wavelet coefficients according to the decomposition technique. Bit error rate (BER) performances of M-ary signals with additive white Gaussian noise (AWGN) through the Rician channel are calculated and graphically shown. The $\mathrm{M}$-ary signal is also used in antenna diversity environment to obtain better BER performance. The simulation study reveals that the proposed technique provides better BER performance of M-ary signal for a multipath fading channel, depending on the weighting control variables. The PDR of the 16-WAM symbol through an AWGN channel for traditional denoising technique is approximately $82.5 \%$ at $E_{b} / N_{0}=3 \mathrm{~dB}$. The proposed technique provides PDR value of $17.3 \%$ of the same symbol through the same channel at the same value of $E_{b} / N_{0}$. Thus the proposed technique provides excellent BER performance along with the antenna diversity technique that can be used in land mobile telephone system in $3 \mathrm{G}$ or $4 \mathrm{G}$ or in WiMAX physical layer to transmit better quality noise free audio or video signal at very high data rate.

The organization of the paper is as follows. In Section 2, the modeling of a multipath fading channel is discussed to characterize the wireless channel. Discrete wavelet transform technique is discussed in Section 3 to generate the wavelet coefficients of a 16-QAM modulated symbol through the fading channel, and wavelet reconstruction is also discussed in this section. Traditional denoising and its complexity for $\mathrm{M}$-ay are discussed in Section 4. The M-ary symbols and its application in wireless signal processing are proposed in

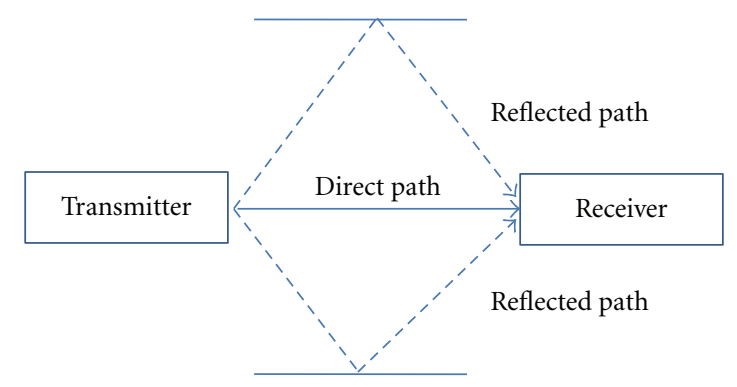

FIGURE 1: Rician fading channel.

Sections 5 and 6. Results of the simulation study are shown in Section 7. Finally, the conclusion of the work is drawn in Section 8 .

\section{Modeling of Wireless Channel}

A QAM bandpass signal $s(t)$ corresponding to the transmitted base-band signals $m_{1}(t)$ and $m_{2}(t)$ with angular frequency $w_{c}$ can be expressed as [19]

$$
s(t)=m(t) e^{(j 2 \pi f c t)}
$$

The result of the signal in (1) transmitted through direct and reflected paths between the transmitter and receiver appears as shown in Figure 1. The reflected paths are the delayed versions of the signal at the receiver, and each path undergoes scattering and the locally scattered and reflected components combine at the receiver, the phenomenon being known as multipath fading. If the direct path of Figure 1 is absent, then the nonline-of-sight (NLOS) paths are characterized by Rayleigh distribution, and the line-of-sight (LOS) propagation paths are characterized by Rician distribution.

The carrier frequency is shifted from the actual one due to the relative movement of either transmitter or receiver or of both. The resultant received signal frequency is given by

$$
f_{r}=f_{0} \pm f_{d}
$$

where $f_{d}$ is the Doppler frequency shift and is defined by

$$
f_{d}=\left(\frac{v}{\lambda}\right) \times \cos \phi
$$

where $\lambda$ is the wavelength, $v$ is the vehicle speed, and $\phi$ is the angle between the direction of wave arrival and the line of the vehicle movement. Due to the effect of Doppler shift, the modulating signal will be [20]

$$
\bar{s}(t)=a e^{j \theta} \exp \left(j 2 \pi f_{d} t\right) m(t) \exp \left(j 2 \pi f_{c} t\right) .
$$

The received signal due to the superposition of say $N$ paths can be written as

$$
\bar{s}(t)=\sum_{k=1}^{N} a_{k} e^{j \theta_{k}} \exp j 2 \pi\left(f_{d, k}+f_{c}\right) t \times m(t),
$$

where, $f_{d k}=(v / \lambda) \cos \phi_{k}, a_{k}$, and $\theta_{k}$ are the attenuation factor and carrier phases. The fading path having a delay 
of $\tau_{k}=h_{k} / c$, where $h_{k}$ is the $k$ th propagation path length and $c$ is the velocity of light. The received signal can be written in the form

$$
\bar{s}(t)=\sum_{k=1}^{N} a_{k} e^{j \theta_{k}} \exp j 2 \pi\left(f_{d, k}+f_{c}\right) t \times m\left(t-\lambda_{k}\right) .
$$

The received signal can be rewritten as

$$
\bar{s}(t)=\int_{-\infty}^{\infty} h(\tau, t) \times s(t-\tau) d \tau,
$$

where

$$
\begin{aligned}
h(\tau, t)= & \sum_{k=1}^{N} a_{k}(t, \tau) \exp \left[j\left(2 \pi f_{d, k} \tau_{k}(t)+\theta_{k}(t, \tau)\right)\right] \\
& \times \delta\left(\tau-\tau_{k}(t)\right), \\
s(t-\tau)= & m(t-\tau) \times \exp j 2 \pi f_{c} t .
\end{aligned}
$$

Hence, $a_{k}(t, \tau)$ is the $k$ th set of complex path gains, $\tau_{k}(t)$ is the excess delay of $k$ th path, and $\delta(\cdot)$ is the delta function. For the Rician channel, the fading process can be written in the form [21]

$$
a_{k}=\sqrt{\Omega_{k}}\left[\frac{z_{k}}{\sqrt{k_{\mathrm{rk}}+1}}+\sqrt{\frac{k_{r k}}{k_{r k}+1}} \times e^{j\left(2 \pi f_{d, k} t+\theta_{k}\right)}\right],
$$

where $k_{\mathrm{rk}}$ is the Rician $k$-factor of the $r$ th path, $\Omega_{k}$ is the average envelope power for $k$ th path. The received complex signal consists of a large number of plane waves as in (7) and the received complex envelope can be written as

$$
\bar{s}(t)=s_{I}(t)+j s_{Q}(t) .
$$

Here, $s_{I}(t)$ and $s_{Q}(t)$ are Gaussian random process with nonzero means $m_{I}(t)$ and $m_{Q}(t)$, respectively. If the random variables $s_{I}(t)$ and $s_{Q}(t)$ have the same variance $r$, then the received complex envelope has a Rician distribution of the form [22]

$$
p(s)=\frac{s}{r^{2}} \times \exp \left[-\left(\frac{s^{2}+z^{2}}{2 r^{2}}\right)\right] \times I_{0}\left(\frac{z s}{r^{2}}\right),
$$

here, $z$ is the direct signal amplitude and $I_{0}(y)$ is the zero order modified Bessel function of the first kind and is defined as

$$
I_{0}(y)=\frac{1}{2 \pi} \int_{0}^{2 \pi} e^{y \cos \theta} d \theta \approx \frac{e^{y}}{\sqrt{2 \pi y}} .
$$

The Rician distribution of the received signal is shown in Figure 2.

\section{Wavelet Transform Algorithm}

The wavelet transform provides the time-frequency representation of the signal through decomposition by passing the time domain signal through various high-pass and low-pass filters. Each filter gives output signal of various frequency bands. The wavelet coefficients after the decomposition proc-

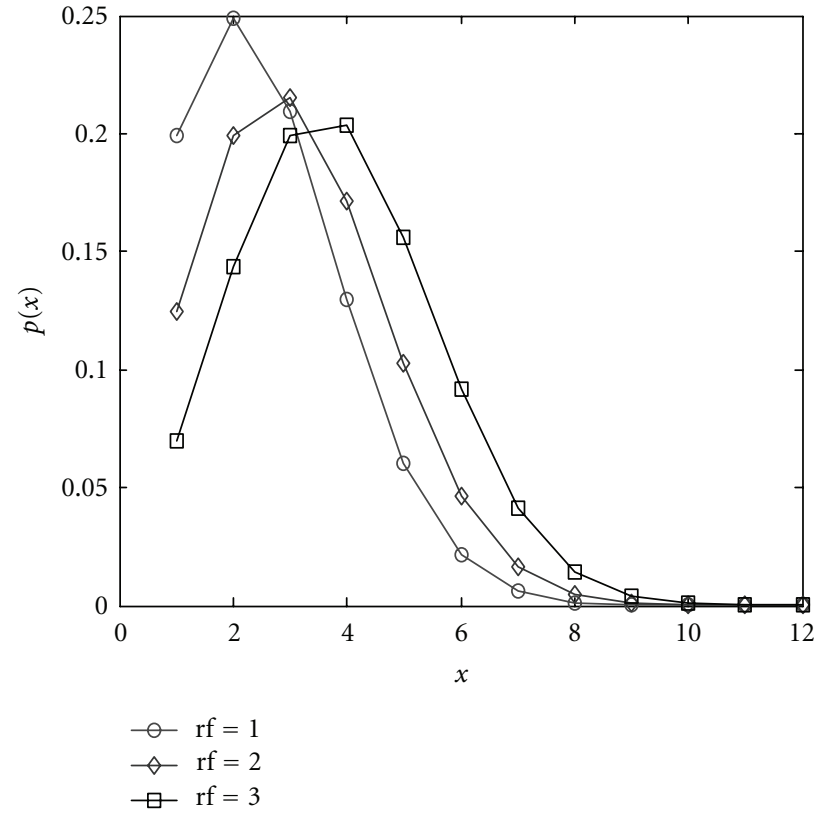

FIGURE 2: pdf of Rician channel and rf represent Rician factor.

ess can be used for further processing to obtain the desired level of signal.

The coefficients are the function of scale and position and are the sum over the signal time multiplied by scale and shifted version of the wavelet function $(\psi)$. Filter technique is used to represent time-scale representation and when the signal passes through filters then its feature is changed by upsampling and downsampling operations. In downsampling operation some of the samples of the signal are removed and in upsampling some of the new samples are added. The wavelet transform is to compute a set of coefficients and each coefficient is the inner product of input function $f(t)$ and basic wavelet $\psi$ [23]:

$$
c(\text { scale, position })=\int_{-\infty}^{-\infty} f(t) \psi(\text { scale, position }) d t .
$$

The continuous wavelet transform is defined [16] as:

$$
c(a, b)=\int_{-\infty}^{\infty} f(t) \overline{\psi_{a, b}(t)} d t,
$$

where $a$ and $b$ represents the scale and position, $\psi_{a, b}$ is defined as

$$
\psi_{a, b}(t)=|a|^{-1 / 2} \psi\left(\frac{t-b}{a}\right) .
$$

The transform becomes

$$
c(a, b)=|a|^{-1 / 2} \int_{-\infty}^{\infty} f(t) \overline{\psi\left(\frac{t-b}{a}\right)} d t .
$$

The discrete wavelet transform can be used to reconstruct the original signal $f(t)$ from the wavelet coefficients [17] as:

$$
f(t)=\frac{1}{c_{\psi}} \iint_{-\infty}^{\infty}|a|^{-1 / 2} \psi\left(\frac{t-b}{a}\right) c(a, b) \frac{d b d a}{a^{2}},
$$

where, $c_{\psi}=2 \pi \int_{-\infty}^{\infty}\left(|\hat{\psi}(\lambda)|^{2} /|\lambda|\right) d \lambda$. 
The family $\left\{\psi_{a, b}\right\}_{(a, b) \in z^{2}}$ of function $\psi$ is an orthonormal basis of the space $L^{2}(R)$ of finite energy signals, the multiresolution analysis (MRA) of $L^{2}(R)$ is a closed sequence $\left\{V_{j}\right\}_{j \in z}$ [18] as

$$
\cdots \subset V_{3} \subset V_{2} \subset V_{1} \subset V_{0} \subset V_{-1} \subset V_{-2} \subset V_{-3} \subset \cdots .
$$

Detail spaces $\left\{W_{j}\right\}_{j \in z}$ are associated with the approximation spaces, and $W_{j}$ is orthogonal complement of $V_{j}$ in $V_{j-1}$ as

$$
V_{j-1}=V_{j} \oplus W_{j} \quad \text { for } j \in z .
$$

As scaling function $\varphi$ is associated with $\psi$, the integer translate of the family $\left\{\varphi_{j, k}\right\}_{k \in z}$ of $\varphi$ generates $V_{j}$. Similarly, the integer translate of the family $\left\{\psi_{j, k}\right\}_{k \in z}$ of $\psi$ generates $W_{j}$. By replacing $j$ by $j-1$, we can decompose $V_{j}$ into its components as

$$
\begin{aligned}
V_{j} & =V_{0}+W_{1}+W_{2}+\cdots+W_{j-1} \\
& =V_{j-1}+W_{j-1},
\end{aligned}
$$

where $V_{j-1}$ and $W_{j-1}$ can be written as [17]

$$
\begin{aligned}
V_{j-1} & =\sum_{k \in z} a_{k}^{j-1} \varphi\left(2^{j-1} x-k\right), \\
W_{j-1} & =\sum_{k \in z} b_{k}^{j-1} \psi\left(2^{j-1} x-k\right),
\end{aligned}
$$

with

$$
b_{k}^{j-1}=\frac{a_{2 k}^{j}-a_{2 k+1}^{j}}{2}, \quad a_{k}^{j-1}=\frac{a_{2 k}^{j}+a_{2 k+1}^{j}}{2} .
$$

Let us consider two discrete filters $H$ and $L$ with their impulse response, $h=(-1 / 2,1 / 2)$ and $l=(1 / 2,1 / 2)$.

Now let us define the following equations:

$$
\begin{gathered}
H(a)_{k}=(h * a)_{k}=\sum_{j=1}^{2} h(j) a_{k+1-j}=-\frac{1}{2} a_{k}+\frac{1}{2} a_{k-1}, \\
L(a)_{k}=(l * a)_{k}=\sum_{j=1}^{2} l(j) a_{k+1-j}=\frac{1}{2} a_{k}+\frac{1}{2} a_{k-1} .
\end{gathered}
$$

Now, replace $k$ by $k+1$, then we have

$$
\begin{gathered}
H(a)_{k}=\frac{1}{2} a_{k}-\frac{1}{2} a_{k+1}, \\
L(a)_{k}=\frac{1}{2} a_{k}+\frac{1}{2} a_{k+1}, \\
H(a)_{2 k}=\frac{1}{2} a_{2 k}-\frac{1}{2} a_{2 k+1}, \\
L(a)_{2 k}=\frac{1}{2} a_{2 k}+\frac{1}{2} a_{2 k+1} .
\end{gathered}
$$

The discarding of odd coefficient is called downsampling, and the decomposition algorithm is shown in Figure 3.

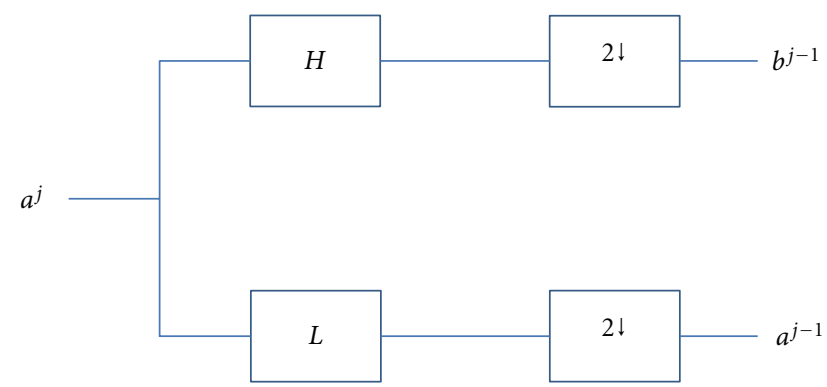

FIgURE 3: Decomposition of $a^{j}$ with downsampling represented by $2 \downarrow$.

Similarly, the reconstruction of $a^{j}$ can be written as

$$
a_{l}^{j}= \begin{cases}a_{k}^{j-1}+b_{k}^{j-1}, & \text { if } l=2 k, \\ a_{k}^{j-1}-b_{k}^{j-1}, & \text { if } l=2 k+1 .\end{cases}
$$

Now, considering the same discrete filters $\widetilde{H}$ and $\widetilde{L}$ with impulse responses $\tilde{h}=(1,-1)$, and $\tilde{l}=(1,1)$, we have

$$
(\tilde{h} * a)_{k}=a_{k}-a_{k-1}, \quad(\tilde{l} * a)_{k}=a_{k}+a_{k-1} .
$$

Setting odd entries to zero, we have

$$
\begin{aligned}
& (\tilde{h} * a)_{l}= \begin{cases}a_{2 k}, & l=2 k, \\
-a_{2 k}, & l=2 k+1 .\end{cases} \\
& (\tilde{l} * b)_{l}= \begin{cases}b_{2 k}, & l=2 k, \\
b_{2 k}, & l=2 k+1 .\end{cases}
\end{aligned}
$$

We have,

$$
(\tilde{h} * a)_{l}+(\tilde{l} * b)_{l}= \begin{cases}a_{2 k}+b_{2 k}, & l=2 k \\ b_{2 k}-a_{2 k}, & l=2 k+1 .\end{cases}
$$

Now, writing $a_{2 k}=a_{k}^{j-1}$, and $b_{2 k}=b_{k}^{j-1}$, the sequence $a_{2 k}$ and $b_{2 k}$ are called upsample and the reconstruction formula is as:

$$
a^{j}=\tilde{L} \text { upsample }\left(a^{j-1}\right)+\tilde{H} \text { upsample }\left(b^{j-1}\right) .
$$

The reconstruction step is shown in Figure 4 .

Multiresolution analysis analyze the signal in different frequency band and enables to observe signal in both time and frequency domain. The three level multiresolution analysis of $a^{j}$ is shown in Figure 5.

The analysis gives the three detail coefficients $c D_{1}, c D_{2}$, and $c D_{3}$, and the approximate coefficient $c A_{3}$. Here $c D_{2}$ is the half resolution of $c D_{1}, c D_{3}$ is the half resolution of $c D_{2}$. 


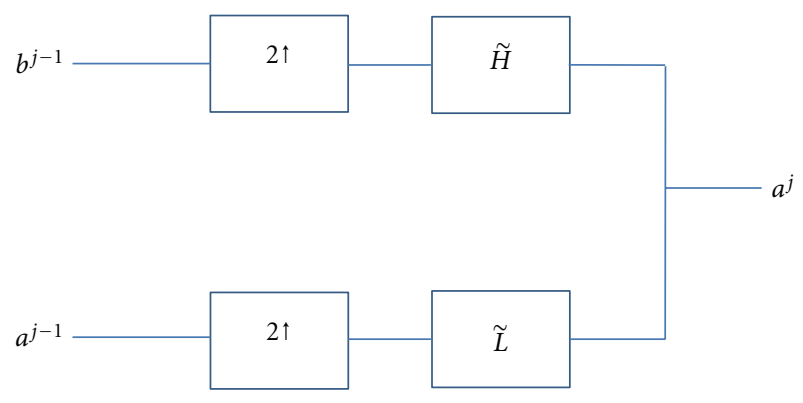

FIGURE 4: Wavelet reconstruction.

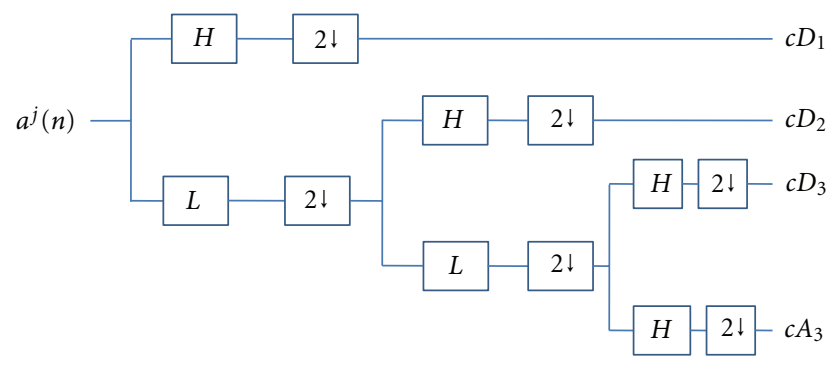

FIGURE 5: Wavelet multiresolution analysis.

These give the detail information of the signal, and the three levels multiresolution step can be written mathematically as

$$
\begin{aligned}
& c D_{1}=\left(H * a^{j}\right) \mid n=2 k, \\
& c D_{2}=L *\left[\left(H * a^{j}\right) \mid n=2 k, k \geq 0\right] \mid n=2 k, \\
& c D_{3}=L *\left\{L *\left[\left(H * \mathrm{a}^{j}\right) \mid n=2 k, k \geq 0\right] \mid n=2 k, k \geq 0\right\} \mid \\
& n=2 k \text {, } \\
& c A_{3}=L *\left\{L *\left[\left(L * a^{j}\right) \mid n=2 k, k \geq 0\right] \mid n=2 k, k \geq 0\right\} \mid \\
& n=2 k \text {, }
\end{aligned}
$$

where $k \geq 0$ in all cases. The multiresolution wavelet reconstruction algorithm is shown in Figure 6.

The multiresolution reconstruction produces final three details $\left(D_{1}, D_{2}\right.$, and $\left.D_{3}\right)$, and approximation $A_{3}$. Mathematically $D_{1}, D_{2}, D_{3}$, and $A_{3}$ can be written as

$$
\begin{gathered}
D_{1}=\left(\tilde{H} * U c D_{1}\right), \\
D_{2}=\left[\tilde{H} * U\left(\tilde{L} * U c D_{2}\right)\right], \\
D_{3}=\left[\tilde{H} * U\left\{\tilde{L} *\left(U c A_{3}\right)\right\}\right], \\
A_{3}=\left[\tilde{L} * U\left\{\tilde{L} *\left(U c A_{3}\right)\right\}\right],
\end{gathered}
$$

where $U a(n)$ is the upsample of $a(n)$ defined as

$$
X(2 n-1)=a(n), \quad x(2 n)=0 .
$$

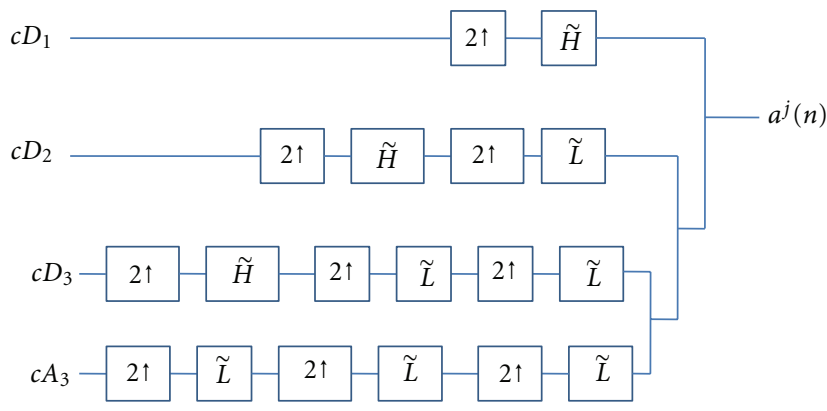

FIGURE 6: Multiresolution reconstruction.

The reconstructed signal can be written as

$$
\overline{a^{j}}(n)=D_{1}+D_{2}+D_{3}+A_{3} .
$$

\section{Problem Statement}

Wavelet denoising is used to predict approximate detail coefficients by allowing minimum error between the detailed coefficients of threshold noisy signal and the original. A noisy random signal is represented as

$$
y(n)=s(n)+\sigma(n),
$$

where $s(n)$ is the original signal and $\sigma(n)$ is the noise with mean zero. Now, decompose the noisy signal $y(n)$ using three levels wavelet decomposition to generate three detail coefficients and one approximate coefficient. A threshold value is applied to each detail coefficient to allow the actual value of the noisy detail coefficient if it greater than the threshold and force to zero otherwise, mathematically can be written as [7]

$$
D_{j, k}^{\prime}=\left\{\begin{array}{l}
D_{j, k} \geq T_{j}, \\
0, \text { otherwise, }
\end{array}\right.
$$

where $T_{j}$ is the threshold value of level $j$. In first stage the threshold can be chosen by finding the minimum error between the detail of noisy and original signal. Later, a loop is applied for each level to find the corresponding threshold value that gives the minimum error. The noisy signal $y(n)$ is denoised by using the threshold level to each detail coefficients after applying three level wavelet transform to generate three detail coefficients. Finally the signal is regenerated using the wavelet reconstruction technique. The result of the denoising of the noisy signal is shown in Figure 7.

In the denoising technique, let the detail coefficients have two levels $a$ and $b$ as in Figure 8(a). When noise affects the signal then the maximum and minimum level may be shifted due to addition of the noise. The threshold level $\mathrm{c}$ is used to allow the coefficient equal to $\mathrm{b}$ when the noisy coefficient is equal or greater than $c$, and equal to a if the noisy coefficient less than c. If the coefficients have multilevel for M-ary modulated signal, that is, another two level $\mathrm{d}$ and e beyond 

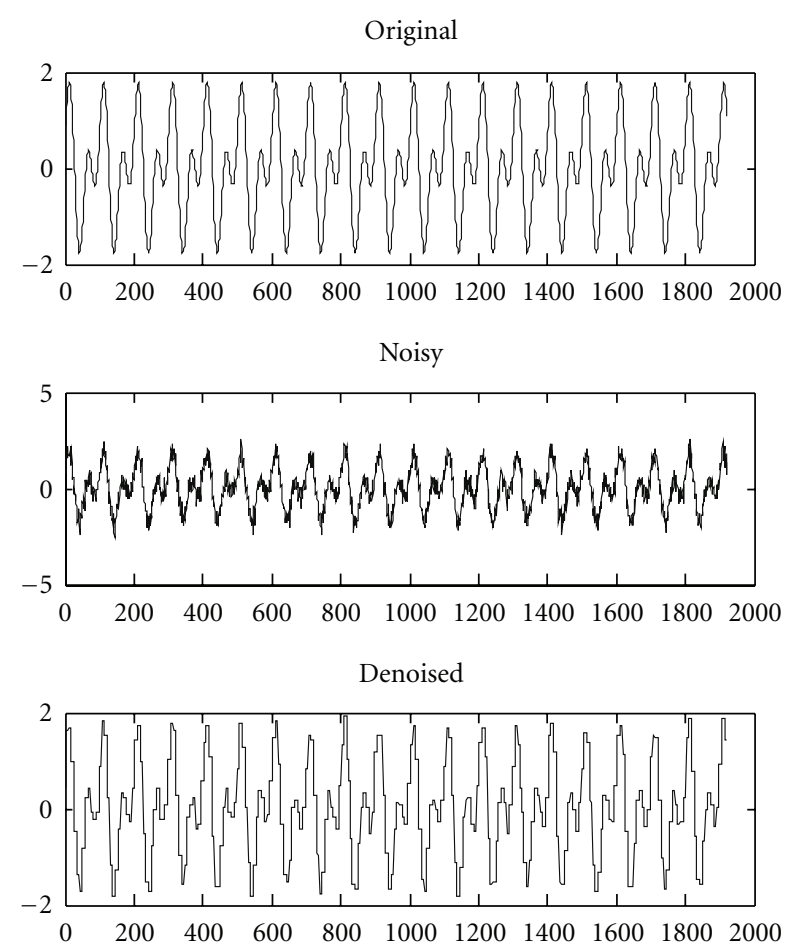

Figure 7: Wavelet denoising of a signal $s(t)$.

$\mathrm{a}$ and $\mathrm{b}$ then the denoising technique is unable to reconstruct the original signal because if the noisy coefficient is less than $\mathrm{c}$ and $\mathrm{a}$ then the technique forces the coefficient equal to a instead of $d$ as shown in Figure 8(b).

\section{Proposed Multilevel Denoising Technique}

A modulated signal $s(t)$ (where $s=a+j b$ ) is affected by noise and interference $n(t)$ (where $n= \pm n_{1} \pm j n_{2}, n_{1}$ and $n_{2}$ are any real value), and the resultant signal can be written as

$$
y(t)=s(t) \pm n(t)
$$

The mean of the white noise is zero, and the mean of the each detail wavelet coefficient is zero [8]. The standard deviation of detail coefficients $\left(c D_{i}\right)$ of $s(t)$ at level $j(j=1,2, \ldots, p$, $p \geq 0 ; p$ is decomposition level) is as

$$
s_{j}=\left(\frac{1}{N-1} \sum_{i=1}^{N}\left(c D_{j i}-\bar{x}_{j}\right)^{2}\right)^{1 / 2},
$$

where $N$ is the length of the detail coefficients, and mean $\bar{x}$ is defined as

$$
\bar{x}_{j}=\frac{1}{N} \sum_{i=1}^{N} c D_{j i}
$$

The standard deviation of detail coefficients of the noisy signal $y(t)$ can be written as

$$
\bar{s}_{j}=\left(\frac{1}{N-1} \sum_{i=1}^{N}\left(\left(c D_{j i} \pm e_{j i}\right)-\bar{x}_{j}^{\prime}\right)^{2}\right)^{1 / 2},
$$

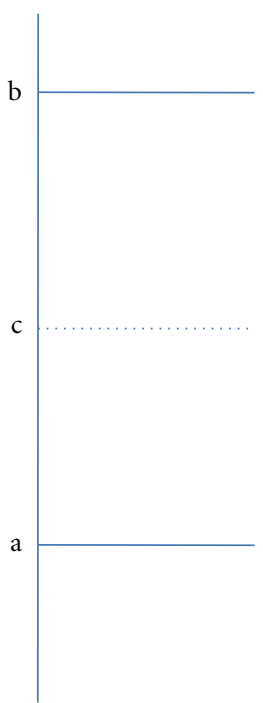

(a)

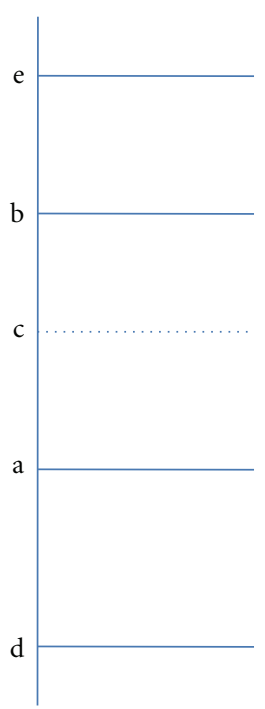

(b)
Figure 8: Wavelet coefficient and threshold level.

where $e_{j i}$ is the components added with the original detail coefficient due the effect of noise. The mean of noisy detail coefficient can be written as

$$
\bar{x}_{j}=\frac{1}{N} \sum_{i=1}^{N}\left(c D_{j i} \pm e_{j i}\right) .
$$

To estimate the original detail coefficients, the mean of noisy detail coefficient $\bar{x}^{\prime}$ should be zero, and the standard deviation can be written as

$$
\bar{s}_{j}=\left(\frac{1}{N-1} \sum_{i=1}^{N}\left(c D_{j i}^{\prime}\right)^{2}\right)^{1 / 2},
$$

where, $c D_{j i}^{\prime}=c D_{j i} \pm e_{j i}$.

A complex weighting vector $w=w_{1}+j w_{2}$, where $w_{1}$ and $w_{2}$ are any real number, is used to minimize random noise by a factor of $w_{1}$ in [15]. In this paper, we used the complex weighting vector to minimize the noise in the detail coefficients by the same amount to generate noise-free wavelet coefficient by approximating the mean of detail coefficient to zero. The modulated signal $s(t)$ is multiplied by a complex variable $w=w_{1}+j w_{2}, w_{1}>0, w_{2}<0$, then the resultant signal can be written as $\bar{y}(t)=s(t) * w$. The noisy received signal can be written as

$$
\begin{gathered}
\bar{y}(t)=s(t) * w \pm n(t), \\
\bar{y}(t)=a w_{1}-b w_{2}+j\left(a w_{2}+b w_{1}\right)+\left( \pm n_{1}\right)+j\left( \pm n_{2}\right),
\end{gathered}
$$

$$
\frac{\bar{y}(t)}{w_{1}}=a-b \frac{w_{2}}{w_{1}}+j\left(\frac{a w_{2}}{w_{1}}+b\right)+\frac{\left( \pm n_{1}\right)}{w_{1}}+j \frac{\left( \pm n_{2}\right)}{w_{1}} .
$$


If $w_{1} \gg w_{2}$, then

$$
\begin{gathered}
y_{\text {out }}(t)=a+j b+\frac{\left( \pm n_{1}\right)}{w_{1}}+j \frac{\left( \pm n_{2}\right)}{w_{1}}, \\
y_{\text {out }}(t)=s(t) \pm \frac{n(t)}{w_{1}} .
\end{gathered}
$$

Here, the noise and interference term in (46) of the received signal $y_{\text {out }}(t)$ through a fading channel is reduced by a factor of $w_{1}$. The mean of the detail coefficients $c D_{j i}$ of less noisy signal $y_{\text {out }}(t)$ in $(46)$ is as

$$
\bar{x}_{j}=\frac{1}{N} \sum_{i=1}^{N}\left(c D_{j i} \pm \frac{e_{j i}}{w_{1}}\right) .
$$

The noise $\left( \pm e_{j i}\right)$ with each of the detail coefficients is due to the noise $n(t)$ with the original signal $s(t)$. The complex number $w$ reduced the noise term $n(t)$ associated with $s(t)$ by a factor of $w_{1}$. If $w_{1} \gg 0$ and $w_{2} \ll 0$ then the mean $\bar{x}_{j} \approx 0$, and the complex number $w$ may be defined as mean control variable. The noise and interference of detail coefficient is reduced by a factor of $w_{1}$, and the reconstructed signal $y_{\text {out }}(t)$ from the noise free detail coefficients may be considered as free from noise and interference as the mean of each detail coefficient tends to zero.

\section{Proposed Denoising Method in Signal Processing}

The noise and interference associated with the wavelet detail coefficient can be discarded using the proposed denoising technique as in (42) to (47). The transmitted signal is multiplied by the complex control variable $w=w_{1}+j w_{2}$. The mean of the detail coefficient is kept at a level approximately equal to zero by reducing the noise term by a factor of $w_{1}$ as according to (46). The value of the mean control variable $w$ is generated according to the required mean value of each detail coefficient. The transmitted signal can be obtained by using wavelet reconstruction as shown in Figure 9. In this figure the modulated symbol $s(t)$ is transmitted through a noisy channel and the noisy signal at the receiver is processed by using three level wavelet decomposition according to (30). The transmitted modulated symbol is reconstructed by using wavelet reconstruction method of the denoised wavelet coefficients according to (31).

Wavelet denoising can be used in antenna diversity technique to obtain better quality signal through a noisy channel. In antenna diversity, signal is transmitted through more than one antenna and received by a single antenna, on the other way one antenna transmits signal and more than one antenna receive the signal. The first process is called transmit diversity and second is called receive diversity. A combiner is used at the receiver to add the entire incoming signal in phase or search the path having highest signal strength [24]. The wavelet denoising method is used to further discarding noise from combiner output signal, the entire process is shown in Figure 10. In transmit diversity, let the signal $S_{0}$ be sent from the transmitter to receiver through two diversity path $h_{1}$ and $h_{2}$, the maximum ratio combiner (MRC) combines the two received fading signal $r_{0}, r_{1}$, according to [24], as

$$
\bar{S}_{0}=\sum_{k=0}^{1} h^{*}{ }_{k} r_{k}
$$

In two branches transmit diversity, symbol from antenna zero can be denoted by $S_{0}$ and from antenna one by $S_{1}$ and in the next symbol period signal the transmitted signal can be represented as $-S_{1}^{*}$, and $S_{0}^{*}$. The received signal for channel response $h_{0}(t)$ and $h_{1}(t)$ can be written as

$$
\begin{aligned}
& r_{0}=h_{0} S_{1}+\left( \pm n_{1}\right)+j\left[h_{0} S_{2}+\left( \pm n_{2}\right)\right] \\
& r_{1}=h_{1} S_{1}+\left( \pm e_{1}\right)+j\left[h_{1} S_{2}+\left( \pm e_{2}\right)\right]
\end{aligned}
$$

here $n_{0}=n_{1}+j n_{2}$, and $e_{0}=e_{1}+j e_{2}$ are the noise signal for 1 st and 2 nd path. Now, we select the control variable of form $w=w_{1}+j w_{2}$, then the resultant transmitted symbol is

$$
S_{0}^{\prime}=S_{0} w=S_{1} w_{1}-S_{2} w_{2}+j\left(S_{1} w_{2}+S_{2} w_{1}\right) .
$$

The received signal can be expressed as

$$
\begin{aligned}
r_{0}^{\prime}= & h_{0} S_{1} w_{1}-h_{0} S_{2} w_{2}+\left( \pm n_{1}\right) \\
& +j\left[h_{0} S_{1} w_{2}+S_{2} w_{1} h_{0}+\left( \pm n_{2}\right)\right] .
\end{aligned}
$$

Dividing (35) by $w_{1}$, and considering $w_{1} \gg w_{2}$, we have

$$
r_{0}^{\prime} \approx h_{0} S_{0}+\frac{1}{w_{1}} n_{0} .
$$

The received signal from other diversity path can be written with applying the same operation as first antenna, we have the received signal as

$$
r_{1}^{\prime} \approx h_{1} S_{0}+\frac{1}{w_{1}} e_{0}
$$

The MRC combiner combined these two received as in (48)

$$
\bar{S}_{0}^{\prime}=\sum_{k=0}^{1} h^{*}{ }_{k} r_{k}^{\prime}
$$

The received signal in (54) is then passed through the wavelet decomposition process to generate the detail coefficients. Here, the control variable $(w)$ is adjusted to approximate the mean of each detail coefficient equal to zero to obtain better channel capacity. The entire process is shown in Figure 10. The PDR is used to examine the percentage of the improvement in the reconstructed signal from the original as

$$
\mathrm{PDR}=\sqrt{\frac{\sum_{n=1}^{N}\left\{V(n)-V_{R}(n)\right\}^{2}}{\sum_{n=1}^{N} V^{2}(n)}},
$$

$V(n)$ : original 16-QAM symbol and $V_{R}(n)$ : reconstructed 16-QAM symbol. 


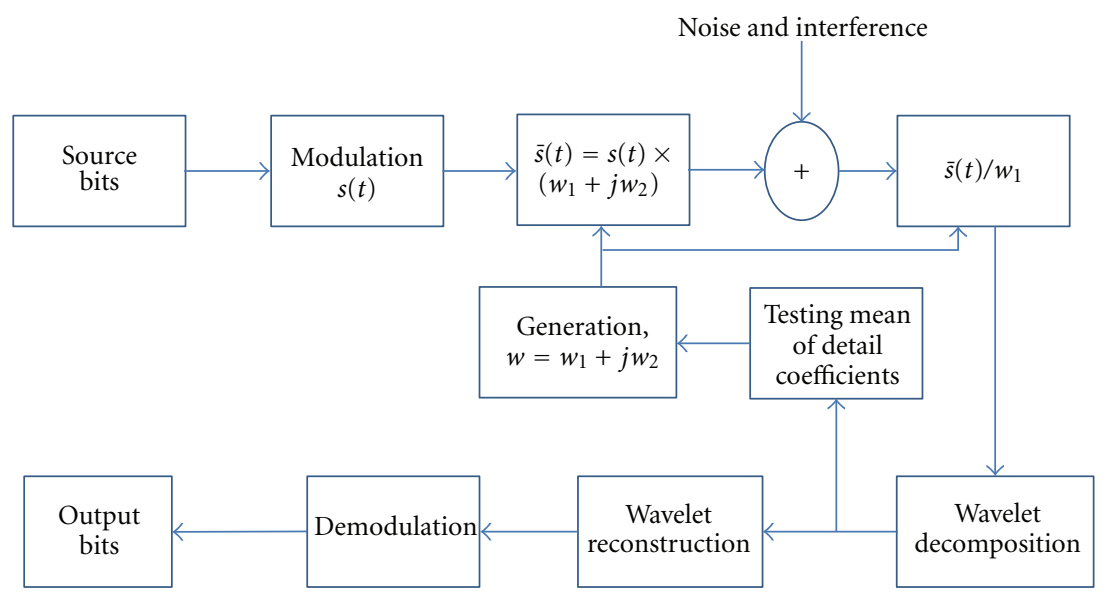

FIgURE 9: Wavelet denoising in communication signal processing.

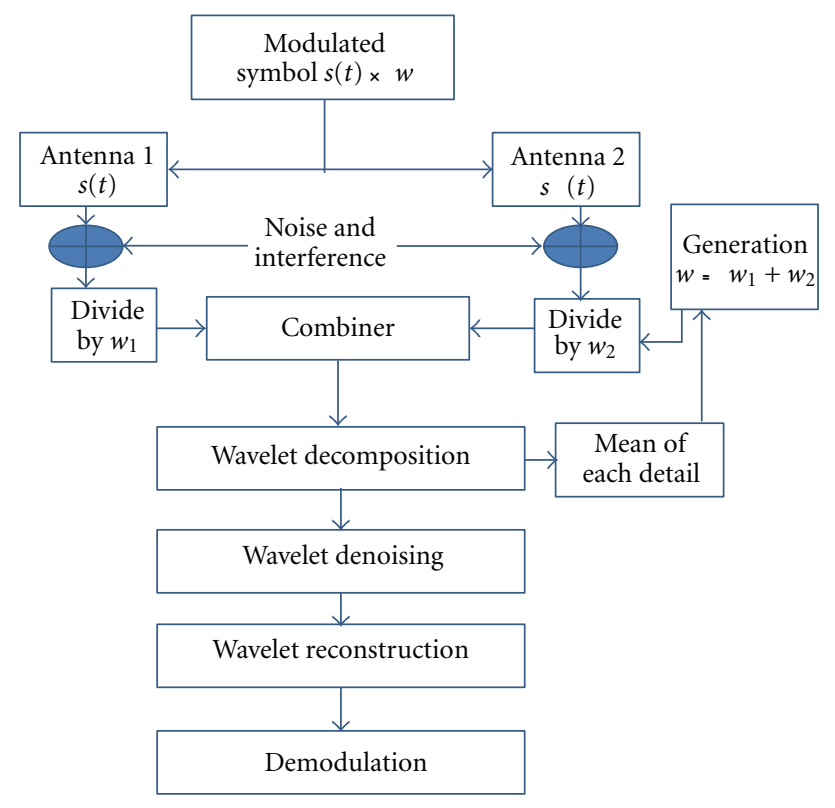

FIGURE 10: Wavelet denoising in antenna diversity.

\section{Results}

The simulation results in MATLAB environment of the BER performance of the AWGN channel for the 16-QAM symbol is shown in Figure 11. Here, the three-level wavelet decomposition technique is applied on the noisy received signal to generate the appropriate detail coefficients. The wavelet reconstruction technique is applied using (31) to generate the detail coefficients which ultimately generate the transmitted signal. The generated transmitted signal is found to be the same as the one received directly from the noisy channel. The multilevel denoising technique shown in Figure 9 is used to increase the performance of the channel. The BER of AWGN channel using different values of the control variable to approximate each detail coefficient equal to zero is shown in Figure 11 for different values of $E_{b} / N_{0}$. The BER through the direct path is 0.2620 at $E_{b} / N_{0}=$

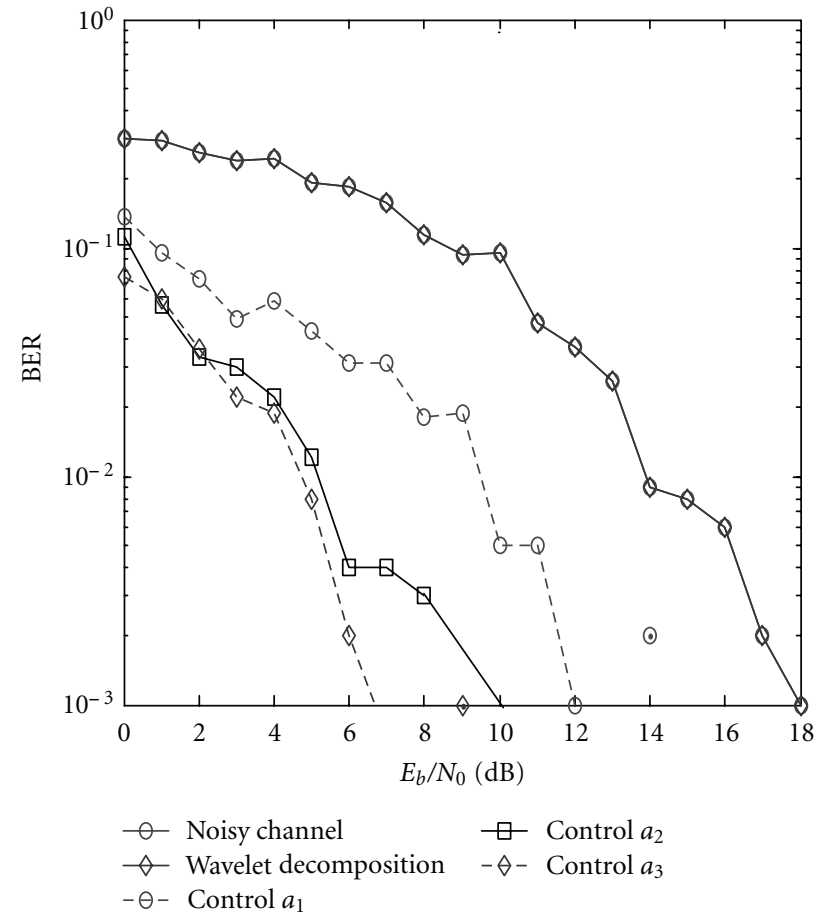

FIGURE 11: BER performance through AWGN channel.

$2 \mathrm{~dB}$ and the wavelet decomposition provides the same BER performance at the same $E_{b} / N_{0}$ value. Now, the controlling value of $1+j 0.2$ is used for which, the BER value is 0.085 for the same parameter. The control variables $a_{1}, a_{2}$, and $a_{3}$ of values of $1+j 0.2,1+j 0.1$, and $1+j 0.001$, respectively, to provide BER values of $0.082,0.0630$, and 0.0290 at $E_{b} / N_{0}$ of $3 \mathrm{~dB}$. The mean of three details coefficients for the above condition is $2.6 \times 10^{-18}$. The control variable allows the mean of the detail coefficients to tend to zero and this phenomenon reduces the noise and interference effects. The BER performance through a Rician fading channel is shown in Figure 12 using the same control variable as of Figure 11 with sampling frequency of $10 \mathrm{MHz}$, Doppler frequency shift of $100 \mathrm{~Hz}$, and the Rician factor of 3. 


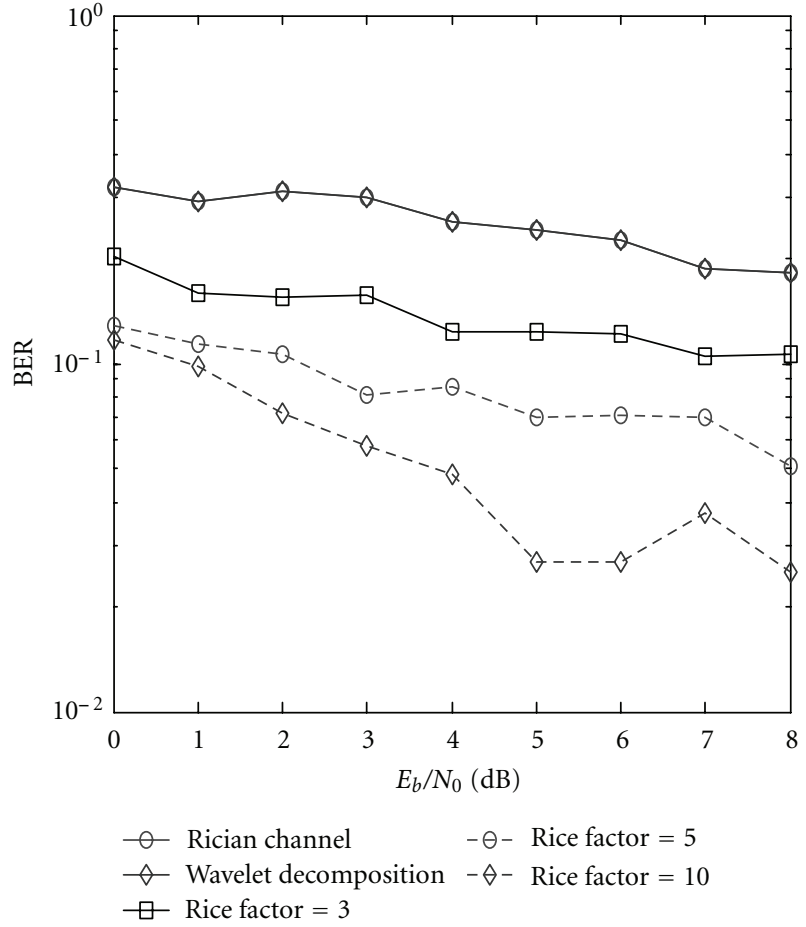

FIGURE 12: BER performance through Rician channel.

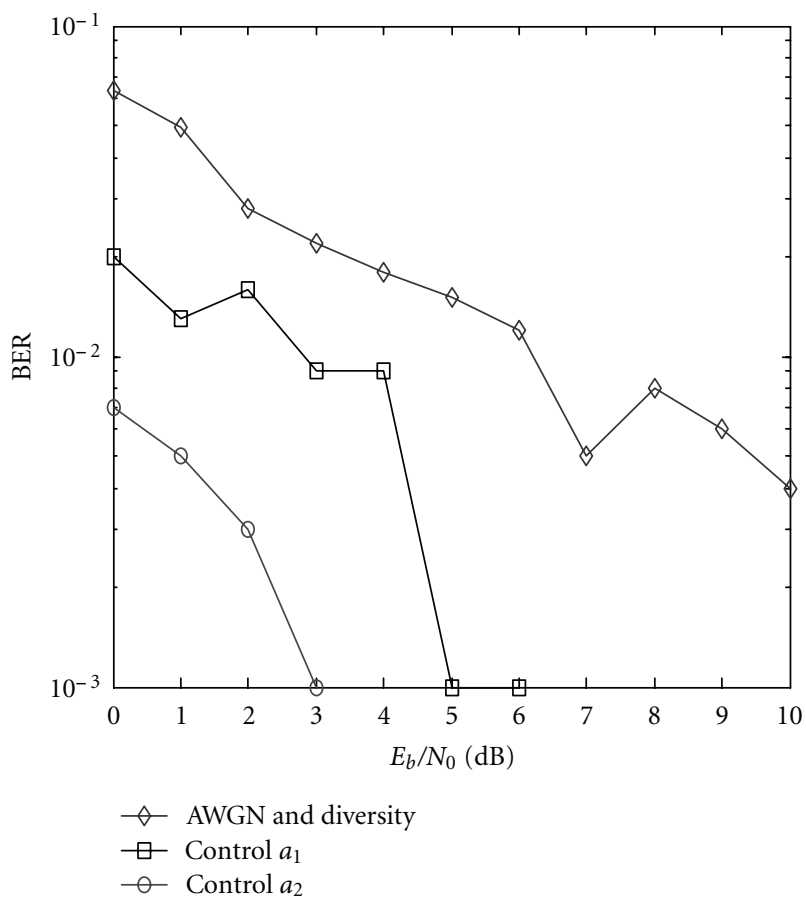

FIGURE 13: BER performance in antenna diversity.

The control variable in antenna diversity is used to reduce the noise level by a factor more than the previous case because diversity provides some noise reduction performance. Here, the BER performance in antenna diversity for the control values of values $a_{1}=2+j 0.01$ and $a_{2}=3+j 0.01$ is shown in Figure 13. The BER is 0.027 for two branch

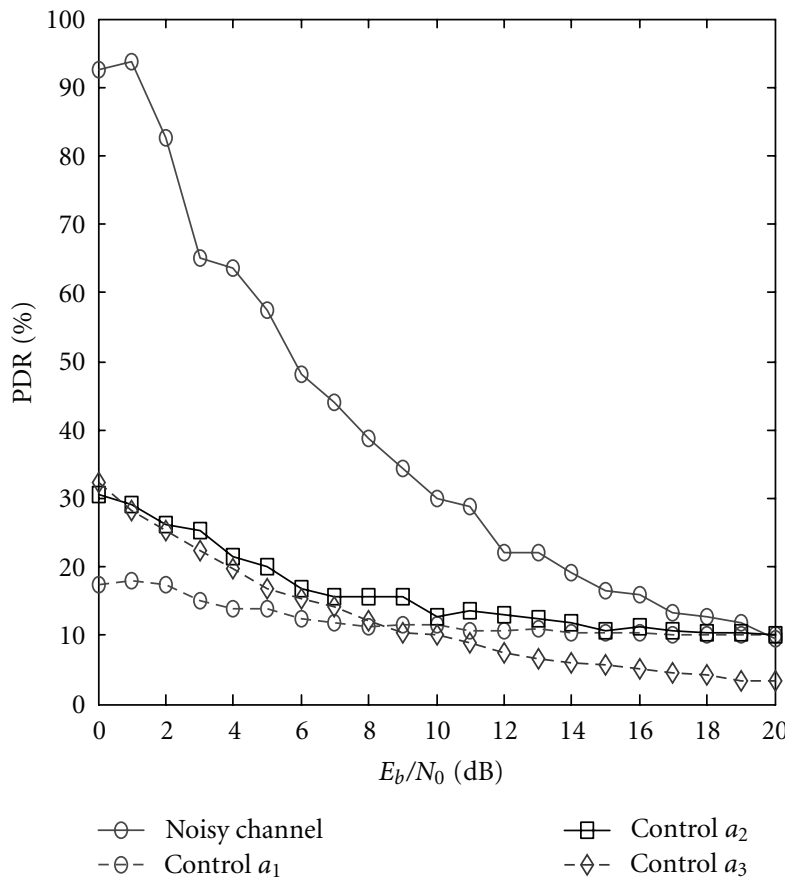

FIGURE 14: PDR of the reconstructed signal through a fading channel.

transmit diversity through the AWGN channel with $E_{b} / N_{0}=$ $4 \mathrm{~dB}$. Now, the BER for the value of control variable $a_{1}=$ $(2+j 0.01)$ and $a_{2}=(3+j 0.01)$ with wavelet denoising in the same branch transmit diversity through the same channel is 0.008 and 0.001 , respectively, with considering the same value of $E_{b} / N_{0}$. Here, the simulation is performed by sending a 16-QAM symbol through an AWGN channel with mean zero and variance $\sigma^{2}$. The phase offsets for the two paths are 30 and 35, respectively. The PDR of the AWGN channel is calculated using (55) by considering $V(n)$ as transmitted 16-QAM symbol. The denoising technique in [7] is used to reconstruct $V_{R}(n)$, and we get the value of PDR which is above $90 \%$ for any value of $E_{b} / N_{0}$. Similarly, the proposed denoising technique is used to reconstruct $V_{R}(n)$ for the same symbol through the same channel. The PDR of the same channel for simply transmitting the symbol through the channel without any signal processing technique, and using proposed denoising technique at different values of control variables is shown in Figure 14 at $E_{b} / N_{0}=0$ $20 \mathrm{~dB}$. It is seen from the result that the proposed denoising technique provides the lowest deviation of reconstruct signal from the original one, because traditional denoising provides PDR of about $92 \%$, direct transmission provides PDR of $82.5 \%$, and the proposed multilevel denoising provides a PDR of $17.3 \%$ for a signal having multipoint in the constellation diagram with $E_{b} / N_{0}=3 \mathrm{~dB}$ for all cases.

\section{Conclusion and Future Work}

Three main issues regarding wireless signal denoising through a multipath fading channel are addressed in this paper. Firstly, the wavelet decomposition and reconstruction 
of a 16-QAM symbol through a noisy channel using MATLAB simulation software. The BER performance of a modulated signal through AWGN and Rician channel and the performance using wavelet denoising technique for the same signal through the same channel is the same as shown from Figures 11 and 12. It is seen from the result that the wavelet decomposition and reconstruction techniques discussed in this paper for the simulation study reconstruct the signal using the wavelet decompose coefficient with $100 \%$ accuracy. Secondly, the traditional threshold-based denoising technique is used to denoise 16-QAM modulated noisy wavelet detail coefficients, the traditional denoising method is not capable to generate noise free coefficients due to the fact that the one threshold level cannot predict a noisy coefficient from multilevel values as discussed in Section 4. Thirdly, a control variable based multilevel wavelet denoising technique is proposed to denoise a complex modulated symbol through a fading channel, and the BER performance is shown using appropriate control variable to force each detail coefficient to zero by reconstructing noise free transmitted symbol.

The simulation result shows that the BER performance is increased with increasing tendency of the mean of the detail coefficients close to zero by selecting appropriate control variable as shown in Figures 11 and 12. The proposed multilevel denoising technique is also used in antenna diversity to obtain a far better BER performance, and the simulation study shows that the proposed denoising technique along with the antenna diversity to provide far better BER performance than the one shown in Figures 11 and 12. The PDR of the proposed multilevel denoising technique shows that it provides accurate reconstruction of a complex modulated symbol through a multipath fading channel because it deviates the estimate signal from the parent one by only $10 \%$.

In the future, the analysis can be done to search for far better a control variable depending on the condition of the wireless channel characteristics to obtain far more accurate results. The proposed technique can be used in multiple input multiple output (MIMO) case to send image signal at very high date rate with far better BER results through WiMAX physical layer.

\section{References}

[1] J. M. Shapiro, "Embedded image coding using zerotrees of wavelet coefficients," IEEE Transactions on Signal Processing, vol. 41, no. 12, pp. 3445-3462, 1993.

[2] H. Choi and R. Baraniuk, "Multiscale texture segmentation using wavelet-domain hidden Markov models," in Proceedings of the 32nd Asilomar Conference on Signals, Systems eramp; Computers, vol. 2, pp. 1692-1697, November 1998.

[3] J. Liu and P. Moulin, "Image denoising based on scale-space mixture modeling of wavelet coefficients," in Proceedings of the International Conference on Image Processing (ICIP '99), pp. 386-390, Kobe, Japan, October 1999.

[4] M. K. Mihçak, I. Kozintsev, K. Ramchandran, and P. Moulin, "Low-complexity image denoising based on statistical modeling of wavelet coefficients," IEEE Signal Processing Letters, vol. 6, no. 12, pp. 300-303, 1999.
[5] J. Portilla, V. Strela, M. J. Wainwright, and E. P. Simoncelli, "Adaptive Wiener denoising using a Gaussian scale mixture model in the wavelet domain," in Proceedings of the IEEE International Conference on Image Processing (ICIP '01), pp. 37-40, October 2001.

[6] L. Sendur and I. W. Selesnick, "Bivariate shrinkage functions for wavelet-based denoising exploiting interscale dependency," IEEE Transactions on Signal Processing, vol. 50, no. 11, pp. 2744-2756, 2002.

[7] M. Alfaouri and K. Daqrouq, "ECG signal denoising by wavelet transform thresholding," American Journal of Applied Sciences, vol. 5, no. 3, pp. 276-281, 2008.

[8] D. Lee Fugal, Conceptual Wavelets in Digital Signal Processing, Space \& Signals Technologies LLC, 2006.

[9] F. Abramovich, T. Sapatinas, and B. W. Silverman, "Wavelet thresholding via a Bayesian approach," Journal of the Royal Statistical Society Series B, vol. 60, no. 4, pp. 725-749, 1998.

[10] A. Chambolle, R. A. DeVore, N. Y. Lee, and B. J. Lucier, "Nonlinear wavelet image processing: variational problems, compression, and noise removal through wavelet shrinkage," IEEE Transactions on Image Processing, vol. 7, no. 3, pp. 319335, 1998.

[11] M. S. Crouse, R. D. Nowak, and R. G. Baraniuk, "Waveletbased statistical signal processing using hidden Markov models," IEEE Transactions on Signal Processing, vol. 46, no. 4, pp. 886-902, 1998.

[12] S. G. Chang, B. Yu, and M. Vetterli, "Adaptive wavelet thresholding for image denoising and compression," IEEE Transactions on Image Processing, vol. 9, no. 9, pp. 1532-1546, 2000.

[13] M. Ghazel, G. H. Freeman, and E. R. Vrscay, "Fractalwavelet image denoising revisited," IEEE Transactions on Image Processing, vol. 15, no. 9, pp. 2669-2675, 2006.

[14] S. Sardy, P. Tseng, and A. G. Bruce, "Robust wavelet denoising," IEEE Transactions on Signal Processing, vol. 49, no. 6, pp. 1146-1152, 2001.

[15] Md. Z. Alam, C. Chittaranjon Patra, C. Patra, and M. Abdus Sobhan, "Modeling and performance analysis of a wireless fading channel," in Proceedings of the 2nd ISECS International Coloquium on Computing, Communication, Control, and Management (CCCM '09), Sanya, China, August 2009.

[16] L. Debnath and D. Bhatta, Integral Transform and Their Application, Chapman \& Hall/CRC, 2nd edition, 2007.

[17] A. Boggess and F. J. Narcowich, A First Course in Wavelet with Fourier Analysis, Prentice Hall, Upper Saddle River, NJ, USA, 2001.

[18] G. Oppenheim, Wavelets and Their Applications, ISTE Ltd., London, UK, 1st edition, 2007.

[19] J. G. Proakis, Digital Communication, McGraw Hill, New York, NY, USA, 3rd edition, 1995.

[20] H. Schulze and C. Luders, Theory and Applications of OFDM and CDMA-Wideband Wireless Comunications, John Wiley \& Sons, London, UK, 2005.

[21] M. C. Jeruchim, P. Balaban, and K. S. Shanmugan, Simulation of Communication Systems, Kluwer Academic/Plenum, New York, NY, USA, 2nd edition, 2000.

[22] G. K. Stuber, Principles of Mobile Communication, Kluwer Academic Publishers, 2001.

[23] S.-T. Bow, Pattern Recognition and Image Processing, Marcel Dekker, New York, NY, USA, 2nd edition, 2002.

[24] S. M. Alamouti, "A simple transmit diversity technique for wireless communications," IEEE Journal on Selected Areas in Communications, vol. 16, no. 8, pp. 1451-1458, 1998. 

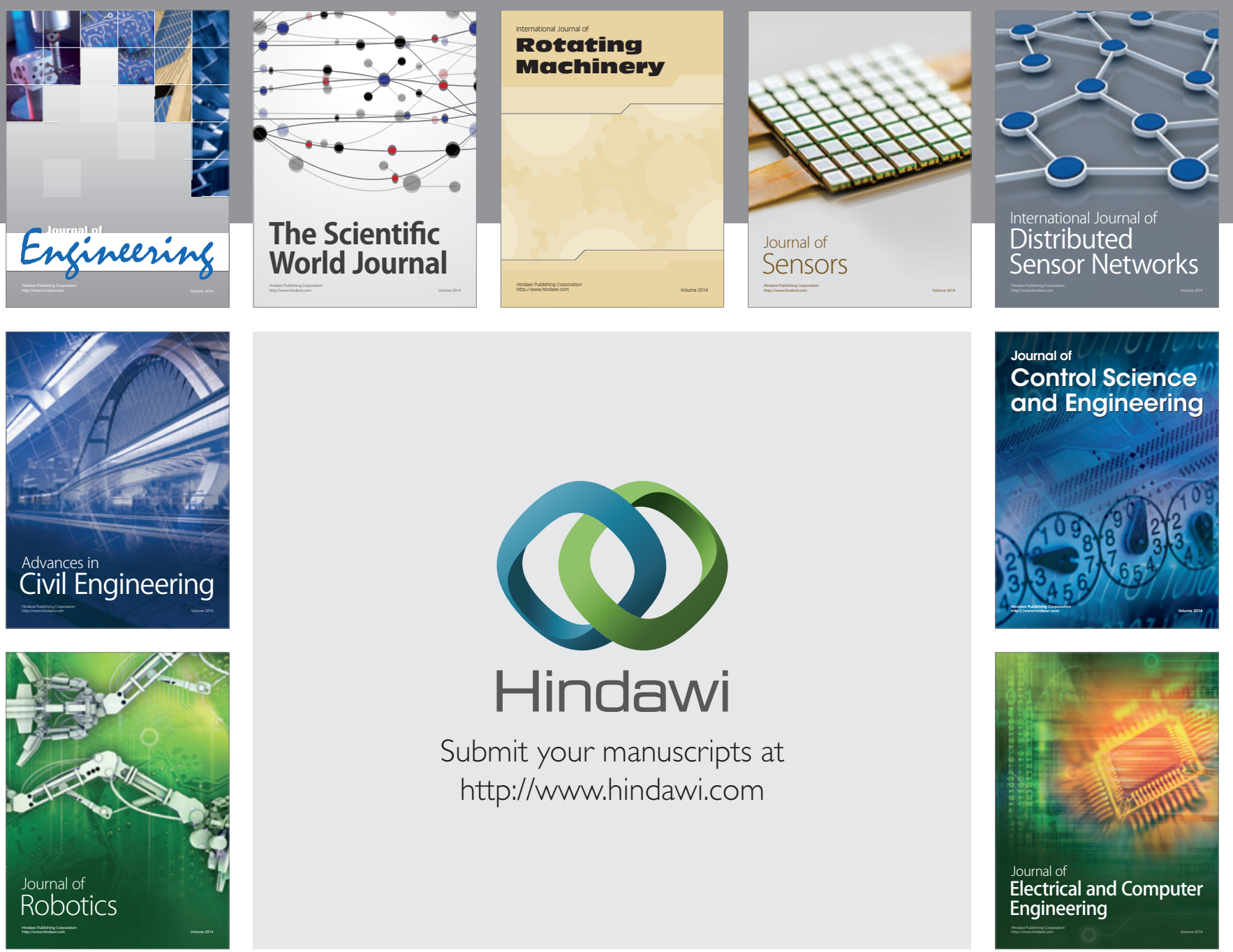

Submit your manuscripts at

http://www.hindawi.com
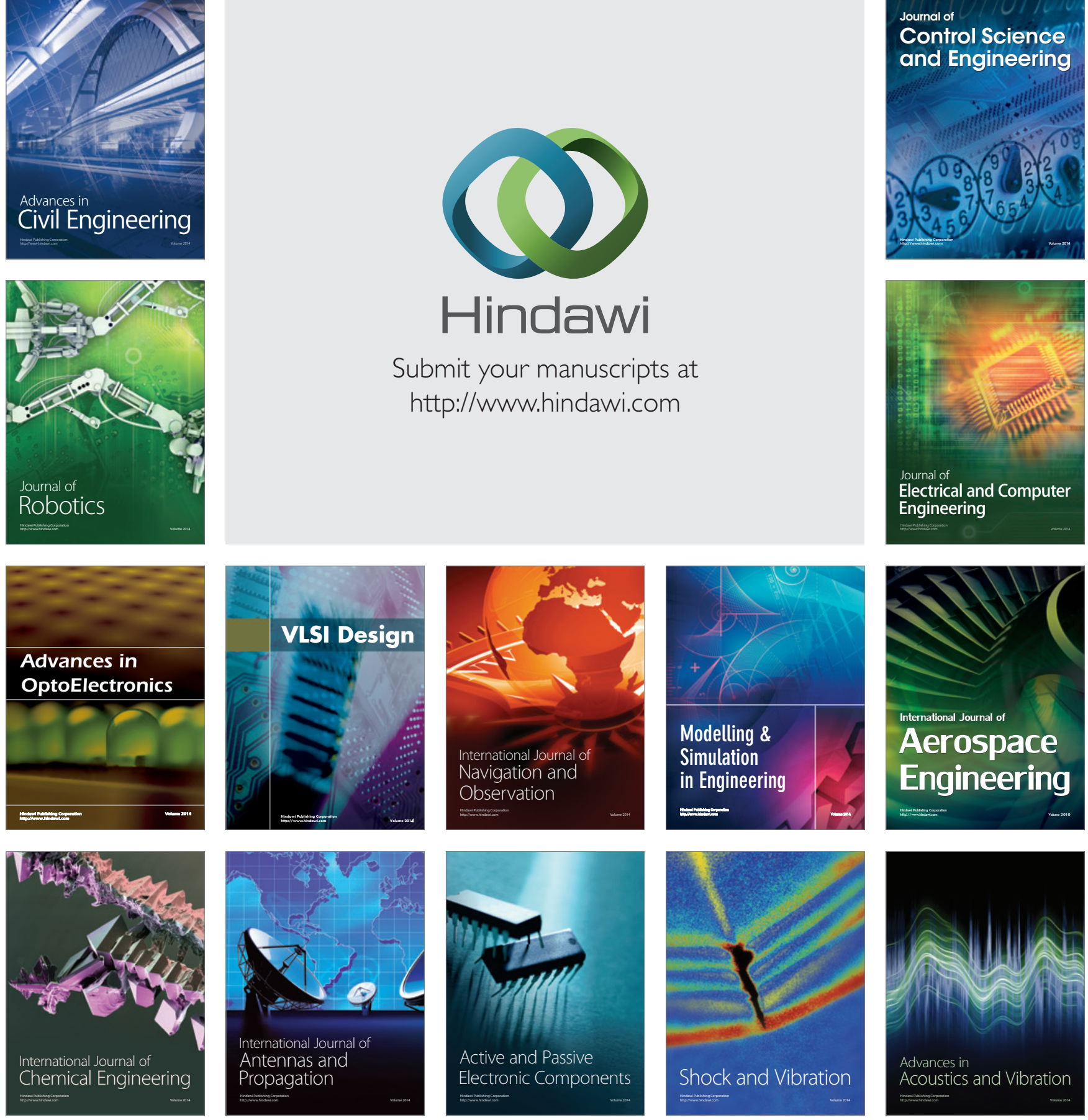